\title{
Board structures around the world: An experimental investigation
}

\author{
ANN B. GILLETTE, THOMAS H. NOE, and MICHAEL J. REBELLO*
}

\begin{abstract}
We model and experimentally examine the board structure-performance relationship. We examine single-tiered boards, two-tiered boards, insider-controlled boards, and outsider-controlled boards. We find that even insider-controlled boards frequently adopt institutionally preferred rather than self-interested policies. Two-tiered boards adopt institutionally preferred policies more frequently, but tend to destroy value by being too conservative, frequently rejecting good projects. Outsidercontrolled single-tiered boards, both when they have multiple insiders and only a single insider, adopt institutionally preferred policies most frequently. In those board designs where the efficient Nash equilibrium produces strictly higher payoffs to all agents than the coalition-proof equilibria, agents tend to select the efficient Nash equilibria.
\end{abstract}

${ }^{*}$ Gillette is on the faculty of Kennesaw State University and the Federal Reserve Bank of Atlanta (e-mail: agillet1@kennesaw.edu), and Noe and Rebello are on the faculty of Tulane University (email: tnoe@tulane.edu and mrebello@tulane.edu). Much of the work on this paper was done while Gillette and Rebello were on the faculty of Georgia State University. We thank participants at the 2005 WFA meetings in Portland, FMA 2004 meetings in New Orleans, the ESA 2003 meetings in Pittsburgh, the Federal Reserve Bank of Atlanta Experimental Finance Conference, the ACLE/JFI Conference On The Ownership Of The Modern Corporation, Stu Gillan, and Mengxin Zhao for helpful comments. Any errors are our own. 


\title{
Board structures around the world: An experimental investigation
}

\begin{abstract}
We model and experimentally examine the board structure-performance relationship. We examine single-tiered boards, two-tiered boards, insider-controlled boards, and outsider-controlled boards. We find that even insider-controlled boards frequently adopt institutionally preferred rather than self-interested policies. Two-tiered boards adopt institutionally preferred policies more frequently, but tend to destroy value by being too conservative, frequently rejecting good projects. Outsidercontrolled single-tiered boards, both when they have multiple insiders and only a single insider, adopt institutionally preferred policies most frequently. In those board designs where the efficient Nash equilibrium produces strictly higher payoffs to all agents than the coalition-proof equilibria, agents tend to select the efficient Nash equilibria.
\end{abstract}




\section{Introduction}

Board structures vary considerably across countries. In the U.S., most boards have majority representation by outside directors and significant insider minority participation. Across the Atlantic, in the U.K., France, and Italy, most boards feature insider majorities with minority outsider participation. Further east, in Germany and Austria, a two-tiered board (dualistisches Model) structure with an insider managerial (Vorstand) board and an outsider supervisory (Aufsichtsrat) board is common.

Recently, following a spate of spectacular corporate failures and scandals, all these board types have been scrutinized by the press, activists, and governments with a view to reforming their structures. In the U.S., NYSE and NASD exchanges have proposed new rules that mandate board independence and tighten the definition of an independent director. The Teachers Insurance and Annuity Association-College Retirement Equities Fund has demanded even stricter standards be imposed. ${ }^{1}$ In the U.K., for the fourth time in the last 10 years, the British government commissioned a new study of board independence (see Economist (January 23, 2003)). In Germany, the federal government established two commissions to examine and suggest improvements to corporate governance practices including the functioning of the two-tiered corporate boards which are pervasive in Germany (PricewaterhouseCoopers (2002)).

The press, government agencies, and investor activists all agree that board reform is desirable. However, they disagree regarding the desiderata for corporate governance reform. Some advocate majority outsider or even supermajority outsider representation. Similarly, some advocate eliminating the dual-tiered boards while still others recommend imposing such a structure on boards. Evaluation of these reform proposals requires an understanding of the relationship between board structure and performance. To obtain this understanding, it is necessary to compare the effectiveness of board structures, which in turn requires controlling for other institutional, economic, and social factors that also affect firm performance. Empirical research is constrained by the lack of independent variation in the control variables and board systems. For example, companies in

1 See corporate Research E-Letter No. 28, October 2002, http://www.goodjobsfirst.org/crp/oct02.htm. 
countries where two-tiered boards are the norm almost always also feature a high-powered large outside investor or lead bank. This makes it difficult to identify the independent effect of the twotiered structure as opposed to large owner monitoring. Also, legal systems are highly correlated with board design, with two-tiered boards concentrated in German civil law countries. Another problem with empirical research on the effects of board structures is the fact that "soft" social factors, which are very difficult to proxy with standard economic variables, may have a huge influence on board performance. In fact, these factors may be even more influential than the formal structure of the board or the legal system in which the firm operates. For example, Franks, Mayer and Rossi (2004) show that despite the lack of any legal protection for minority shareholders in the nineteenth and early twentieth centuries, the capital market in the U.K. developed at a much more rapid pace than its continental counterparts which featured some, albeit weak, protection of outsiders. Franks, Mayer, and Rossi hypothesize that outside investors in the U.K. were protected better by social networks and social norms than outside investors in other countries were by formal legal institutions. ${ }^{2}$ Finally, as noted by Hermalin and Weisbach (2001), board structure is endogenous, and performance affects structure just as board structure affects performance. For example, is a negative relation between board size and performance an indication that large boards make poor decisions or that poorly performing firms expand their boards? As argued by Hermailin and Weisbach, this endogeneity problem makes unambiguous interpretation of empirical results on boards and performance difficult. Moreover, as Coles, Lemmon, and Meschke (2003) point out, the endogeneity problem cannot be corrected with simple econometric fixes but requires estimating a complete structural model of the firm. Thus, any conclusion regarding governance and performance from cross-sectional studies depends for its validity on identifying the correct structural model for the relation between managerial compensation, corporate investment policy, board structure, and ownership dispersion. Using a purely theoretical approach, it is equally difficult to identify the relationship between performance and the structure of communication and voting on boards. Many

2 In addition to these factors board structure designs systematically vary with insider trading law enforcement (see Bhattacharya and Daouk (2002)) and institutional investor activism (Kahn and Winton (1998)). 
different board structures produce identical sets of Nash equilibrium outcomes. Further, in general, many Nash equilibria are supported for each board structure. ${ }^{3}$

The considerable practical importance of comparing board structures, combined with the lack of guidance from conventional theoretical and empirical studies, suggests that researchers examine the board structure-performance relationship from a new direction-experimental study. This is the approach we adopt in this paper. Here we experimentally compare the performance and voting behavior of a variety of board types. To facilitate experimental analysis, and to provide sharp hypotheses in the context of which to view our results, we initiate our analysis by developing a simple model of corporate board decision making. Our model captures the basic tradeoff between inside and outside directors: insiders have better information but outsiders have better incentives. Insiders have private information regarding the quality of a project that the firm can undertake, but are biased toward accepting the project regardless of their information. Outside directors have no private information about project quality but an incentive to block low-quality projects. To emphasize the stylized role of outside directors in our analysis we call outside directors "watchdogs." This model design abstracts from all factors influencing corporate governance other than board structure. Although the absence of these factors reduces the usefulness of the model for making cross-sectional empirical predictions, it increases the usefulness of the model for doing what it is designed to do-isolating the effect of board structure in mitigating opportunistic insider behavior and predicting the results of our laboratory experiments.

Of course, just as field experiments on board structure and performance suffer from obvious econometric problems, so experimental simulation of corporate decision making raises the question of external validity - to what extent do our university-student subjects behave like seasoned executives acting in real boardrooms? Validity can be questioned on two grounds. First, will the much smaller payoffs to lab subjects relative to board members lead to differences in behavior? Sec-

3 The theoretical literature on boards, with one exception, Gillette, Noe, and Rebello (2003), has focused on other issues. For example, Raheja (2004) considers how managerial succession affects board performance; Hermalin and Weisbach (1991) consider managerial bargaining power and how it affects board performance. 
ond, will the different levels of maturity and the contrasting social milieu of business students and board members render our student results uninformative regarding board behavior? Both of these questions are empirical and cannot be answered with a priori reasoning. However, we are fairly confident that the weight of evidence favors external validity. First, consider payment scale differences. There is a considerable body of research on the effect of monetary payoffs and experimental subject behavior, articulated most notably by Smith (1976). For games where computational or memory effort is not an important consideration, as in our game, the evidence this research suggests that shifts in the level of payments received by the agents do not have a dramatic effect on behavior when subjects are paid relative to the opportunity cost of their time. ${ }^{4}$ The second objection is that the personal characteristics of board members and the social context for board decisions is so different from that faced by student classroom subjects, that drawing conclusions from one regarding the other is indefensible. This sort of localist argument is logically defensible but is not consistent with the available scientific evidence or with the conclusions of evolutionary psychology which argues that similar ecologically relevant problems are solved in a roughly similar way by most humans. ${ }^{5}$

4 For example, see Davis and Holt (1992) pp.24-26; Hoffman, McCabe, and Smith (1996); and Forsythe et al. (1991). However, there is evidence that "microscopic" payments, far below the subject's perceived level of fair compensation, can reduce performance even below the zero payoff level in games where the subject is paid for performing tasks (e.g., taking an IQ test) (see Gneezy and Rustichini (2000)).

5 For empirical evidence where professional play has been compared to responses of student subjects in a variety of market contexts, see DeJong et. al. (1988); Dyer, Kagel, and Levin (1989); Mestelman and Feeny (1988); Plott (1988); and Smith, Suchanek, and Williams (1988). These studies find that the behavior of professional decision makers does not qualitatively differ from that exhibited by the student subject group. Furthermore, the positive use of transferability of experimental results to corresponding nonexperimental settings has been discussed, among others by Smith (1982), and in a regulatory context by Issac and Smith (1985) and Plott (1987). The concept of "ecologically relevant problems" is based on the idea that humans solve most practical problems by referring them back to a few heuristic rules, rules that were relevant and adaptive to 
We consider four different board structures: a two-tiered board, a single-tier board with a onevote watchdog majority and multiple insiders, a single-tier board with a one-vote insider majority, and a board with just one insider. We characterize Nash equilibria for these four board types, focusing on their ability to support institutionally preferred corporate policies that maximize firm value and on the nature of the coordination on which they rely. It is plausible to assume that agent coordination will lead to outcomes that reflect the interests of the agents. For this reason we consider two refinements to the Nash equilibrium - efficient Nash equilibria and coalition-proof Nash equilibria. An equilibrium is efficient if no other equilibrium exists that Pareto-dominates that equilibrium. An equilibrium is coalition-proof if no credible coalition can overturn the equilibrium (Bernheim, Peleg, and Whinston (1987)). We first show that regardless of the number of watchdog directors or whether the board has one or two tiers, there does not exist any coalition-proof Nash equilibrium that implements value maximizing corporate policies. Further, despite differences in communication mechanisms, single-tiered boards with watchdog majorities and multiple insiders are equivalent to two-tiered boards in terms of the equilibrium outcomes they support. Both board types implement institutionally preferred corporate policies in an efficient Nash equilibrium. The coalition-proof outcome for both board types is underinvestment. A board with a single tier and an insider majority can also support Nash equilibria supporting institutionally preferred corporate policies. However, these equilibria are not efficient. Further, all coalition-proof equilibrium out-

our ancestors during our species' evolutionary history. Problems that can be referred back to such paradigms, "ecologically relevant problems," will be solved in a roughly similar and reasonable manner by most humans. We argue that the board of directors problem that we model fits into a general collection of ecologically relevant human decision problems, problems whereby some members of a group have both better information and selfish interests, and need to persuade other members of the group to trust their judgment. Such problems were faced by our hunter-gatherer ancestors and are faced on a regular basis today, such as by a tenure committee considering the opinion of faculty members who are expert scholars in the candidate's field, but are also the candidate's personal friends. See, for example, Tooby and Cosmides (1992). The authors would like to thank Peter Bossaerts for directing our attention to this literature. 
comes supported by insider-majority boards result in overinvestment. The single-tiered board with only one insider does not support any equilibria producing institutionally preferred policies. In fact, the only equilibrium outcome supported by this type of board is a coalition-proof equilibrium resulting in underinvestment.

Using these results as our benchmarks, we performed a laboratory experiment on university graduate and undergraduate students. In the experiment, contrary to the coalition-proof refinement, even minority representation of watchdogs reduced opportunistic behavior by insiders and frequently produced value-maximizing outcomes. Thus, even insider majority boards, as long as they feature substantial watchdog participation, can produce value-maximizing policies. Switching to a two-tiered board resulted in a perceptible decrease in opportunistic behavior by insiders and an increase in the frequency with which value-maximizing policies were adopted. However, treatments which featured single-tiered boards with one-vote watchdog majorities produced much better results. In these treatments nearly all (93\%) of the sessions produced value-maximizing outcomes. Reducing the number of insiders further had little effect on the outcome. Although both the two-tiered board and the single-tiered board with an insider majority frequently produced inefficient outcomes, they displayed different biases. The two-tiered board tends toward underinvestment, frequently rejecting good projects, while the single-tiered board tended toward overinvestment, frequently accepting bad projects.

Together with the baseline results in Gillette, Noe, and Rebello (2003), which documents that all-insider boards almost always choose inefficient self-interested policies, our results document that increasing watchdog representation on boards increases efficiency. Moreover, by varying the level of watchdog participation, this paper is able to document that the marginal benefit of watchdog participation, while always positive, is decreasing. Although this decreasing rate of improvement is not always consistent with the predictions of some game-theoretic refinements, it is consistent with empirical research. For example, Klein (2002) finds that increasing the number of independent outsiders on audit committees improves reporting. However, almost all the gains from increasing the number of independent outsiders are largest when the number of insiders was large. Little improvement was generated by increasing outsider representation on committees that were already outsider-dominated. These results are also consistent with the experimental economics literature 
on trust that agents are more likely to display trustworthy behavior when they have less power (see, e.g., Berg, Dickhaut, McCabe (1995)).

The remainder of the paper is organized as follows. Section II describes the game, delineates the equilibria, and describes their properties. Section III describes the experimental design. Section IV presents results from treatments designed to test our theoretical predictions. In Section V, we present evidence from additional treatments. The final section concludes the paper and suggests directions for future research. Proofs are confined to Appendix A.

\section{Model}

\section{A. Agents and Information}

A board consists of $n$ agents belonging to the set $N$. The first $w>0$ are watchdogs who belong in the set $W$. The remaining $i$ agents are insiders from the set $I$. At the beginning of the game, insiders receive an information signal, $s$, revealing whether a project is good $(G)$ or bad $(B)$. Project acceptance increases value if the observed signal is $G$ and destroys value when it is $B$. Watchdogs cannot observe project quality but believe that it is good (bad) with probability $\pi$ $(1-\pi)$.

\section{B. Payoffs}

Insiders' payoffs depend on their signal regarding project quality, whether the project is accepted, and whether a penalty, $P$, is assessed on insiders for a lack of consensus. Let $a \in\{0,1\}$ represent the project acceptance decision with $a=1$ representing project acceptance $(A)$ and $a=0$ indicating rejection $(R)$. Let $c \in\{0,1\}$ represent a lack of consensus among board members, with $c=1$ indicating consensus and $c=0$ indicating a lack of consensus. In the absence of consensus among board members, a penalty may be imposed at random on insiders on the board. Let $z \in\{0,1\}$ indicate the imposition of the penalty, where $z=1$ indicates that insiders are assessed a penalty for lack of consensus. Thus, the ex post payoff of an insider, which we represent by $U_{I}$, is as follows:

$$
U_{I}(a, c, s, z) \equiv a x(I, A, s)+(1-a) x(I, R, s)-P(1-c) z
$$

where $x$ is a function mapping agent identity, voting outcome, and signal into payoffs. If the agent is a watchdog, his payoffs depend only on the insiders' signal regarding project quality and the 
outcome of the vote. Thus, the ex post payoff of a watchdog, which we represent by $U_{W}$, is as follows:

$$
U_{W}(a, s) \equiv a x(W, A, s)+(1-a) x(W, R, s) .
$$

Let $\rho$ equal the probability that the penalty indicator $z=1$. The rankings of the payoffs, $x$, given in the above equations, are as follows:

$$
\begin{gathered}
x(I, A, G)>x(I, R, G), x(I, A, B)>x(I, R, B) ; \\
x(W, A, G)>x(W, R, G), x(W, A, B)<x(W, R, B) . \\
\pi x(W, R, G)+(1-\pi) x(W, R, B)>\pi x(W, A, G)+(1-\pi) x(W, A, B) . \\
\rho x(I, A, s)-\rho P>x(I, R, s), s=G \text { or } B .
\end{gathered}
$$

Assumption 3 ensures that project acceptance is preferable to insiders regardless of project quality and that watchdogs prefer to accept the project only when it is good. Assumption 4 implies that, if watchdogs have to make their accept/reject decision based on their prior information, they prefer to reject the project. Assumption 5 implies that insiders are willing to pay the price for lack of consensus if, by paying this price, they will ensure acceptance of the project.

\section{Actions and Strategies}

The board members play a game consisting of two stages: (1) a communication stage and (2) a decision stage. Communication occurs after insiders receive their information signal. It is "cheap" and has no direct effect on agent welfare. Let the $j$ th watchdog's communication strategy be represented by the message $m_{j}^{W} \in M^{W}$, where $M^{W}$ is a message space. Similarly, let insider $j$ 's communication strategy be given by the message $m_{j}^{I} \in M^{I}$.

After board members exchange messages, they vote on the project. Insiders can either vote to accept the project, $\mathcal{Y}$, or to reject it, $\mathcal{N}$. Watchdogs can either vote to reject, $\mathcal{N}$, or abstain from voting, $\mathcal{A}$. Let $v=\left(v_{1}, \ldots v_{N}\right)$ represent the vector of board votes, $V$ represent the set of all possible vote vectors, $v^{W}$ represent the subvector of watchdog votes, and let $v^{I}$ represent the subvector of insider votes. For any vector (or subvector of) $v$, let $\# \mathcal{Y}(v)$ represent the number of yes votes, $\# \mathcal{A}(v)$ represent the number of abstentions, and let $\# \mathcal{N}(v)$ represent the number of no votes. 
The board structure determines the rules for project acceptance and the definition of consensus. In single-tiered boards, all board members cast their votes simultaneously. The project is accepted if strictly more votes are cast for the project than against the project. Let $a^{O}$ indicate project acceptance for a single- (or one-) tiered board. It follows that $a^{O}(v)=1$ if and only if $\# \mathcal{Y}(v)>\# \mathcal{N}(v)$. Let $c^{O}$ be the indicator function for consensus on a single-tiered board. That is, $c^{O}(v)=1$, if and only if $\# \mathcal{Y}(v)>\# \mathcal{N}(v) \Rightarrow \# \mathcal{N}\left(v^{I}\right)=0$ and $\# \mathcal{Y}(v) \leq \# \mathcal{N}(v) \Rightarrow \# \mathcal{Y}\left(v^{I}\right)=0$.

A two-tiered board consists of one board that only includes insiders and another that only includes watchdogs. Each of the two boards meets separately following the revelation of the project signal to the insiders. The insider board casts the initial vote. If a majority of insiders vote to reject the project, the project is not undertaken and no vote is required of the second or watchdog board. Otherwise, the project's fate is decided by a majority vote by the watchdog board. The project is accepted unless a majority of the watchdog board vote to reject. Let $a^{T}$ indicate project acceptance for a two-tiered board. It follows that $a^{T}(v)=1$ if and only if $\# \mathcal{Y}\left(v^{I}\right)>\# \mathcal{N}\left(v^{I}\right)$ and $\# \mathcal{A}\left(v^{W}\right) \geq \# \mathcal{N}\left(v^{W}\right)$. Insiders face a penalty for lack of concensus if either there is a split vote on the insider board or if unanimous acceptance by the insider board is followed by rejection by the watchdog board. Thus, $c^{T}$, the indicator function for consensus on a two-tiered board, equals 0 if and only if $0<\# \mathcal{Y}\left(v^{I}\right)<i$, or $\# \mathcal{Y}\left(v^{I}\right)=i$ and $\# \mathcal{N}\left(v^{W}\right)>\# \mathcal{A}\left(v^{W}\right)$.

\section{Results}

In this section we develop the hypotheses that are tested using our experimental data. We examine voting patterns of boards and the investment policies adopted by them. In our context, board effectiveness is captured by the nature of the investment policy adopted by the board. The first-best policy, which we term the institutionally preferred policy, calls for project acceptance if and only if project quality is good, that is, when insiders observe signal $G$. First, we examine voting by single-tier boards. We examine three types of single-tier boards-(i) a board with an insider majority, as is the case with most boards in countries such as the U.K. and France; (ii) a board with a single insider and watchdog majority as has been advocated by a number of commentators; (iii) finally, we examine voting when watchdogs command a majority on the board and the board has

multiple insiders as is the case with most U.S. boards. The section concludes with an examination of two-tiered boards that are common in countries such as Germany and the Netherlands. 


\section{E. Single-tiered Boards}

Consider a board with a majority of insiders. Every insider prefers undertaking the project to rejecting it regardless of her information signal. Because insiders command a majority on the board, watchdogs are powerless to overturn any insider consensus. Thus, insiders can force acceptance of the project if they act in concert. Further, a defection by any group of insiders that can result in project rejection is not sustainable, as a subgroup of the defectors will always be able to increase their payoffs by defecting back to voting for project acceptance regardless of its quality. It follows that effective coordination between insiders can lead to overinvestment by insider-dominated boards.

Theorem 1. In all coalition-proof equilibria, when $i>w$, all insiders vote to accept the project under both information signals, watchdog votes are unrestricted, and the project is accepted regardless of the information signal. Moreover, coalition-proof equilibria exist.

Proof. See Appendix.

Insider dominated boards can also implement the institutionally preferred policy. This is likely to occur when coordination between insiders is not possible or breaks down. With multiple insiders on the board, insiders know that if other insiders are providing "honest" recommendations by conditioning their messages on the information signal and voting in accordance with their recommendation, unilateral efforts of a single insider to ensure project acceptance under both signals is futile and will simply call down the penalty for consensus failure. Because the implementation of the institutionally preferred investment policy produces the highest possible payoff to watchdogs, they will vote in a manner that will not counter the insider votes. Thus, an equilibrium in which all insiders truthfully report their signals is sustainable and results in implementation of the institutionally preferred policy. We call this outcome, consensus among insiders and truthful revelation of their information resulting in the implementation of the institutionally preferred policy, the efficient outcome.

Theorem 2. (i) When $i>w$, there exists a Nash equilibrium implementing the efficient outcome. In all Nash equilibria that implement the efficient outcome all insiders vote $\mathcal{Y}$ if they observe the signal $G$ and vote $\mathcal{N}$ if they observe the signal $B$.

Proof. See Appendix. 
A switch to a watchdog majority board tilts the balance of voting power away from insiders. This can render insider coordination futile. If the shift in voting power is accompanied by reducing insider representation to one, the tilt toward watchdogs also results in a dramatic change in board effectiveness. Because there is but a single insider on the board, insider coordination is perfect. This eliminates the possibility of efficient equilibria that result in the institutionally preferred policy. To see this, note that for the institutionally preferred policy to be implemented it would have to be the case that the insider sends messages that accurately reveal his signal regarding project quality. If watchdogs vote in accordance with the insider's messages, he can always achieve his most preferred outcome, project acceptance regardless of the information signal, by switching the message he issues following the receipt of the signal $B$ to the message he transmits following the receipt of $G$.

Theorem 3. (i) When $w>i=1$, there does not exist any Nash equilibrium implementing the efficient outcome.

In addition to eliminating efficient equilibria, a switch to a watchdog majority with only one insider also changes the nature of the coalition-proof equilibria. Because the watchdogs cannot rely on the solitary insider to accurately reveal his information signal, they are left with the choice of either always voting to reject the project or always voting to accept. Because they prefer rejecting to accepting based on their prior beliefs, watchdogs will choose the latter course of action. Because there is but one insider on the board, only one watchdog need always cast a reject vote while the rest abstain. Faced with watchdog rejection of the project, to avoid incurring the penalty for board dissent, the insider will also always vote to reject regardless of his signal.

Theorem 4. In all coalition-proof equilibria, when $w>i=1$, the insider votes to reject the project under both information signals, at least one watchdog also votes to reject, and the project is rejected regardless of the information signal. Moreover, coalition-proof equilibria exist.

Proof. See Appendix.

If the watchdog majority is maintained and multiplicity of insiders is restored, we arrive at the situation examined in Gillette, Noe, and Rebello (2003). As they demonstrate, coordination among the watchdog majority eliminates the possibility of overinvestment resulting from coordination among insiders. Thus, all coalition-proof equilibria result in underinvestment. However, for the 
reasons described earlier, the multiplicity of insiders ensures that, when they do not coordinate their actions, Nash equilibria exist that implement the institutionally preferred investment policy.

Theorem 5. When $w>i>1$, (i) In all coalition-proof equilibria, (a) all insiders vote to reject the project under both information signals and (b) under both information signals, enough watchdogs vote against the project to ensure that, even if all insiders were to switch their votes to acceptance, the project would still be rejected. Moreover, coalition-proof equilibria exist. (ii) A Nash equilibrium implementing the efficient outcome exists. In all Nash equilibria that implement the efficient outcome all insiders vote $\mathcal{Y}$ if they observe the signal $G$ and vote $\mathcal{N}$ if they observe the signal $B$.

Proof. See Appendix.

Together, these results indicate that the composition of a single-tiered board can have a marked impact on the nature of decisions made by the board. A multiplicity of insiders is essential for the implementation of the institutionally preferred policy. Further, the direction of any distortion from the institutionally preferred policy is crucially dependent on the identity of the group commanding the majority of votes on the board - a watchdog majority biases investment policy toward underinvestment while an insider majority biases it toward overinvestment.

\section{F. Two-tiered Boards}

Now consider a two-tiered board. With a two-tiered board, watchdogs control one board tier and can block insider proposals even if they are outnumbered by insiders overall. Because watchdogs have effective blocking power in two-tiered boards, even when they are outnumbered by insiders, they have the ability to force outcomes that are consistent with the presence of a watchdog majority on a single-tier board. Further, because there are many insiders, just as in the case of a single-tiered board with multiple insiders, no one insider can change the outcome of the vote when other insiders are voting together. As explained earlier, this permits Nash equilibria that implement the institutionally preferred policy. In summary, when there is more than one insider on the board, the theoretically predicted outcomes for two-tiered boards and single-tiered boards with watchdog majorities are identical.

Theorem 6. On a two-tiered board, when $i>1$, (i) In all coalition-proof equilibria, all insiders vote to reject the project under both information signals. (ii) A Nash equilibrium implementing 
the efficient outcome exists. In all Nash equilibria that implement the efficient outcome all insiders vote $\mathcal{Y}$ if they observe the signal $G$ and vote $\mathcal{N}$ if they observe the signal $B$.

Proof. See Appendix.

In summary, when insiders control the board, it is in their collective interest to form coalitions that produce overinvestment. Once insiders lose control, either through becoming a minority on a single-tiered board or having their vote subjected to a veto by a second board tier consisting of watchdogs, the formation of insider coalitions is counterproductive for insiders themselves. In this case, the coordination problem produced by including many insiders on the board supports equilibria which are preferred by both insiders and watchdogs.

\section{Experimental Design}

We ran an experiment to examine the performance of each of the board types examined above. The experimental subjects were undergraduate and graduate students. They were told they would have an opportunity to earn money in a research experiment involving group decision making. Every subject participated in only one experimental session.

\section{A. The Basic Design}

In the following section we describe the experimental procedures for all treatments. All the single-tiered board treatments employed identical procedures. ${ }^{6}$ The two-tiered board treatments employed similar procedures with the necessary modifications made to accommodate the additional tier of the board. We first describe the procedures for the single-tiered board. We then outline the differences in the instructions and procedures for the two-tiered board.

Once subjects were assembled at the venue for an experiment, they were read a set of instructions (see Appendix B); they completed assigned worksheets, and were given the opportunity to ask questions. The language used throughout the experiment was neutral with respect to the framing of the problem. At the end of the instructional period, the monitors randomly assigned subjects their agent type - insider or watchdog, and then assigned them to groups of seven.

6 The experimental procedures for the single-tiered board treatments are similar to those employed by Gillette, Noe, and Rebello (2003) except that we provide subjects with higher payoffs. 
Groups dispersed to different ends of a large classroom to commence the session. Each round began with a period of communication between group members. Communication followed the group-subgroup sequence: Communication was permitted first among all members of a group, and then within subgroups based on agent type. The following restrictions applied: No physical threats, no side payments, no communication among groups, and a maximum of four minutes for each discussion period. Subjects never appeared to find this time limit to be binding. Next, the insiders from each group watched as one of them was randomly chosen to draw the project type from a bucket. To ensure that good and bad draws had equal probabilities, the bucket contained 50 white chips (good outcome) and 50 red chips (bad outcome) and chips were replaced after each draw. Following a draw, the insiders returned to their groups and a discussion among all members of each group commenced. The discussions seldom approached the two minute time limit. Private ballots were cast following this discussion. A monitor then counted the votes. The project was undertaken if the yes votes outnumbered the no votes. When the yes votes equaled or were fewer than the no votes, the project was rejected. The monitor privately informed each group of the outcome and distribution of votes by agent types.

All single-tiered board treatments incorporated the following penalty feature for split votes: If at least one insider's vote did not conform with the majority vote for the group, a monitor drew a chip from a bucket of poker chips that contained 20 blue chips and 80 white ones. Chips were replaced after each draw. All insiders in the group were assessed a penalty if a blue chip was drawn. The outcome, together with the project type and the occurrence of a split vote, determined payoffs for the round.

In accordance with Assumption 3, payoffs were designed to ensure that insiders preferred to accept the project regardless of the outcome. Absent the penalty for lack of consensus, they received at least $\$ 2.70$ following project acceptance, compared with a maximum $\$ 1.80$ following its rejection. When a penalty was imposed, insider payoffs fell by $\$ 0.75$. Thus, insiders earned at least $\$ 1.95$ if the project was undertaken even if a penalty was imposed. Because this was higher than their expected $\$ 1.80$ payoff if the project was rejected and no penalty was imposed, in accordance with Assumption 5, the penalty for lack of consensus was not sufficient to reverse their preferences between investing in the project and rejecting it. Watchdogs' payoffs were designed to ensure that 
they preferred taking on the project only if it was good. They could expect to earn $\$ 2.10$ from project acceptance conditional on a good draw and $\$ 0.30$ from acceptance conditional on a bad draw. Consistent with Assumption 4, their expected payoff of $\$ 1.20$ from acceptance was less than their expected payoff of $\$ 1.50$ from rejection. All payoffs were common knowledge.

A round ended after subjects learned about the outcome, participated in the penalty draw if applicable, and calculated their earnings. Each experimental session consisted of 10 rounds, but subjects essentially played a game with an indefinite endpoint since they were not told how many rounds they would play.

The two-tiered board treatments differed in terms of the communication and voting procedures as well as the rules for determining the outcome of the vote and the imposition of a penalty draw. In the two-tiered treatments, subjects were assigned to one of two boards - the managerial board consisting of four members and the supervisory board consisting of three members. ${ }^{7}$ All insiders were assigned to the managerial board and all watchdogs were assigned to the supervisory board. First, as in the case of the single-tiered board, insiders on the managerial board met as a subgroup and then observed the outcome of the project quality draw. Each insider then voted to either accept or reject the project. If the majority of the managerial board voted to reject the project, the round ended. Otherwise, a representative of the managerial board conveyed the voting distribution to the supervisory board. After receiving this information and having an opportunity to question the representative of the managerial board, members of the supervisory board privately voted to either reject the project or abstain. A project recommended by the managerial board was considered to be accepted so long as no more than one member of the three-person supervisory board voted to reject. A penalty draw was imposed on the insiders under two conditions-(i) a split vote by the

7 Care was taken to ensure that the language employed in the experiments was not suggestive. We chose to use this board composition rather than one in which the managerial board had three members and the supervisory board four members. Our choice was made to highlight the fact, explained in the previous section, that watchdogs do not need to outnumber insiders to gain voting control of a two-tiered board. Further, all two-tiered boards with multiple insiders are theoretically equivalent in that they support the same equilibrium outcomes. 
managerial board or (ii) unanimous approval by the managerial board followed by rejection by the supervisory board. The payoff structure for subjects was identical to the structure used in the single-tiered board treatments; that is, subject payoffs contingent on the project quality draw, the board decision, and the penalty draw were identical across the single-tiered and two-tiered board treatments.

For each type of board we employed two treatments - a random mixing protocol (RAN) whereby, after each round, subjects were randomly assigned to new groups, and for robustness a repeated mixing protocol $(\mathrm{RE})$ where subjects stayed in the same groups for the entire experimental session. In all treatments, subjects maintained their agent type for the entire session. This variation in mixing protocols was motivated by extant research, which demonstrates that mixing protocols can influence experimental outcomes (see, e.g., Eckel and Holt (1989)). The random mixing protocol is designed to implement the single-shot games modeled in the previous section. Thus, we would expect stronger correspondence between the results in random mixing treatments and our theoretical predictions. The repeated mixing protocol allows us to examine the robustness of our results to repeated interactions between board members, which is common in actual boards.

Table I describes the salient features of the treatments including the number of groups employing each treatment. The first four columns describe the random mixing treatments. The last four columns describe the repeated treatments. The treatment WMAJRAN modeled single-tiered, watchdog-controlled boards and used four watchdogs and three insiders. The treatment labeled IMAJRAN was designed to examine single-tiered insider-controlled boards and employed four insiders and three watchdogs, while in the treatment labeled SIRAN only one subject was assigned the role of the insider and the other six subjects were assigned to be watchdogs on a single-tiered board. The two-tiered random mixing treatment is labeled TTRAN. A separate treatment employing the repeated groups protocol was employed for each of these board structures. The single-tiered majority watchdog treatment with repeated groups is labeled WMAJRE, its insider majority counterpart is labeled IMAJRE, and the single-tiered single-insider treatment with repeated groups is labeled SIRE. The two-tiered repeated mixing treatment is labeled TTRE.

\section{Results}

We now examine results from the random mixing treatments that most closely fit our the- 
oretical analysis along four dimensions:(1) the incidence of the institutionally preferred outcome, (2) insider voting patterns, (3) watchdog voting patterns, and (4) the predictive success of the two competing equilibria. The results indicate that single-tiered watchdog majority boards are most efficient in implementing the institutionally preferred policy. Two-tiered boards are more efficient than single-tiered boards with insider majorities. However, they display a conservative bias and are more likely than single-tiered boards to reject good projects. The results also indicate that the efficient equilibria have greater predictive power for single-tiered boards with watchdog majorities and for two-tiered boards. In contrast, the coalition-proof equilibria supporting overinvestment perform better when single-tiered boards have insider majorities.

\section{A. Incidence of the Institutionally Preferred Outcome}

Table II presents the frequency with which the institutionally preferred outcome occurred. From the table it is apparent that single-tiered boards with watchdog majorities are the most successful in implementing the institutionally preferred outcome. The institutionally preferred outcome was implemented at least 93.3 percent of the time in these treatments. Single-tiered treatments employing multiple insiders (WMAJRAN) generated results very similar to those from treatments employing only a single insider (SIRAN). Two-tiered boards were less effective in implementing the institutionally preferred policy. It was implemented 80 percent of the time (TTRAN). Single-tiered boards with an insider majority resulted in the lowest incidence of the institutionally preferred outcome. It occurred 61.7 percent of the time (IMAJRAN). A noteworthy difference between the performance of the two-tiered boards and the single-tiered boards is that two-tiered boards resulted in a higher incidence of rejection of good projects. The relative efficiency of the two-tiered boards in comparison to the single-tiered board with an insider majority arises from the higher rejection rate for bad projects. These two results suggest that two-tiered boards are more conservative than single-tiered boards.

Chi-square tests reported in Table III confirm that the incidence of the institutionally preferred outcome is significantly lower when there is a majority of insiders on the board relative to when there is a majority of watchdogs on the board. This difference is more pronounced following bad draws. Table III also confirms our interpretation of the differences between two-tiered boards and single-tiered boards. The tests suggest that, for good projects, the likelihood of the implementation 
of the institutionally preferred policy by a two-tiered board will be significantly different from the likelihood of implementation by any of the three forms of single-tiered boards examined. For bad projects, the difference in the likelihood of implementation of the institutionally preferred policy is only significant for two-tiered boards versus single-tiered boards with insider majorities. In order to control for unobserved heterogeneity caused by differences in the characteristics of the participants in the different sessions, we also tested for differences in aggregate outcomes using a random effects probit model. These results are reported in Table IV. Except in cases such as the single insider treatment (SIRAN) where all rounds and all sessions produced the same outcome (making it impossible to distinguish between session and treatment effects) these results are consistent with those of the simple Chi-square tests reported in Table III.

These experimental results contrast in a number of dimensions with our theoretical predictions. First, insider majority boards do implement the institutionally preferred outcome most of the time. In fact, even after a bad draw, when insider interests are opposed to institutional interest, they implement the institutionally preferred outcome in excess of 15 percent of the time. This result contrasts strongly with our theoretical results which show that insiders obtain a uniformly higher payoff in the coalition-proof equilibria in which the project is always accepted. Another interesting contrast between theory and the experimental outcomes is the efficiency advantage of single-tiered watchdog majority boards over two-tiered boards. Both board designs support identical coalitionproof outcomes as well as identical efficient outcomes. Finally, in strong contrast to the predictions of even the Nash equilibrium concept is the efficiency of the single-insider single-tiered board. Under this design, the efficient outcome can never be produced by a Nash equilibrium. Nevertheless, this design had nearly equivalent efficiency with the most efficient board structure design in the experiments - the single-tiered watchdog majority. At the same time, we obtain many results that are consistent with theory. Notably, passing from a single-tiered insider majority board to a two-tiered board increases efficiency. Also, although the efficiency of the insider majority board is higher than expected given theory, consistent with theory, it is less efficient than single-tiered watchdog majority and two-tiered designs.

\section{B. Insider Voting Patterns}

Table V presents the distribution of insider votes across the four treatments. Insiders tended 
to vote as a group. The only exceptions were one round of treatment WMAJRAN, two rounds of treatment IMAJRAN, and four rounds of TTRAN. In the single-tiered board treatments all instances of split insider votes followed bad draws for project quality. Following a good draw all insiders in all groups across all treatments voted to accept the project except in two rounds of TTRAN. Following bad draws, there is evidence that some insiders voted to accept in every board design with the exception of the single insider board. For example, all insiders voted to accept 4 of 33 times in treatment WMAJRAN. However, this voting pattern was common only in the single-tiered insider majority treatments and two-tiered treatments.

Overall, insider voting patterns varied across treatments as one would expect given the variation in the implementation of the institutionally preferred outcome. Single-tiered boards with insider majorities generated the highest frequency of yes votes by insiders following bad draws. While such votes were frequently observed in the two-tiered board treatments, their incidence was less frequent. The lowest frequency of yes votes by insiders following bad draws was observed in the single-tiered board treatments with watchdog majorities. The frequency of unanimous no votes by insiders following bad draws tells a similar story regarding opportunistic behavior of insiders. In the IMAJRAN sessions, only in 3 of 28 rounds of bad draws did all of the insiders in a group vote against the project. In comparison, the number of unanimous no votes by insiders climbed to 19 out of 34 in treatment TTRAN, 28 out of 33 for treatment WMAJRAN, and 20 out of 20 in treatment SIRAN.

\section{Watchdog Voting Patterns}

Watchdog voting patterns are presented in Table VI. With the exception of the single-tiered insider majority treatment, watchdog votes appear correlated with the outcome of the project quality draw - watchdogs tended to abstain following good draws and vote to reject following bad draws. This suggests that insiders were accurately transmitting the outcome of the project quality draw. However, there are many deviations from this overall voting pattern across all treatments. Further, there are also a number of instances in which watchdog votes were split. Split votes were cast 37 of 60 times, the highest frequency of such votes in treatment IMAJRAN, the board design that provides the insiders with the greatest opportunity to induce the acceptance of bad projects because of their dominance on the board. The lowest frequency of split votes occurred in the 
single-tiered board treatments that included a majority of watchdogs. These results, together with the earlier evidence on insider voting patterns, suggest that the increased incidence of opportunistic behavior by insiders is associated with lower consensus among watchdogs.

\section{Subject Votes and Equilibrium Vote Vectors}

In this section we examine the predictive power of the equilibria described earlier. To help assess the results of the experiments, we first describe the predictions of our model for parameter values that are equal to those employed in our experiments.

Theorem 1 demonstrates that coalition-proof equilibria result in overinvestment when boards have a majority of insiders. For boards with four insiders and three watchdogs, Theorem 1 suggests that, in all coalition-proof equilibria, all four insiders vote to accept the project under both information signals, while Theorem 2 indicates that, in all efficient equilibria, all four insiders vote for the project under information signal $G$ and against the project under information signal $B$. In neither case do the equilibrium requirements restrict watchdog votes. A switch to a majority watchdog board changes both the outcome of coalition-proof equilibria and equilibrium voting patterns. When the board includes only one insider, Theorem 3 predicts that the sole insider will vote against the project regardless of the information signal, as will at least one watchdog. If there are three insiders and four watchdogs on the board, Theorem 5 suggests that, in all coalition-proof equilibria, all three insiders and at least three watchdogs vote to reject the project under both information signals, while all three insiders vote for the project under information signal $G$ and against the project under information signal $B$; and at least two watchdogs abstain when the information signal is $G$ in all efficient equilibria. Theorem 6 predicts that, in all efficient equilibria supported by a two-tiered board, all members of the insider board vote to reject the project when the information signal is $B$ and accept the project when the information signal is $G$. At least two members of the supervisory board will abstain when the signal is $G$. Further, in all coalition-proof equilibria, all insiders on the managerial board always vote to reject the project regardless of their information signal.

To examine the predictive power of the competing equilibria, we computed Selten's (1991) measure of predictive success for each equilibrium for each treatment. This measure of predictive success is computed as $\rho-\alpha$, where $\rho$ is the relative frequency with which outcomes conform 
with the given equilibrium and measures the accuracy of the theory. The second term, $\alpha$, is the probability that outcomes consistent with the equilibrium occur by random chance and captures the precision of the theory's predictions. The results are reported in Table VII.

In line with our discussion of the observed frequency of outcomes consistent with the competing equilibria, we find that the efficient equilibria enjoy greater predictive success than coalition-proof equilibria for two-tiered boards and single-tiered watchdog majority boards. The difference is primarily attributable to the poor performance of the coalition-proof equilibria following good draws. Coalition-proof equilibria also perform poorly following good draws with single-tiered single insider boards but enjoy much greater predictive success in the treatments with single-tiered insider majority boards, where the overall success rate for coalition-proof equilibria is 0.85 compared with 0.52 for efficient equilibria. The high observed success rate following bad draws, predicted by coalition-proof equilibria, is primarily responsible for the high predictive success of these equilibria.

Given that the unique Nash equilibrium outcome for single-tiered boards with a single insider call for no cooperation or information sharing between insiders and watchdogs, this high level of efficiency is surprising. However, this result is not surprising from the perspective of experimental research. In our experiments, revalation of the insiders' information always leads to efficient outcomes whenever watchdogs command a majority. Such information sharing between subjects, even when information sharing is not in the subjects' self-interest is pervasive in experiments. (see, e.g., Valley, Thompson, Gibbons, and Bazerman (2002)). Even in contexts where efficient outcomes cannot be achieved by simple information sharing, agents frequently coordinate to efficient non-Nash outcomes (see, e.g., Ledyard (1995), Camerer (2003, and Fehr, Kircksteiger, and Reidl (1993)). For example, Berg, Dickhaut, and McCabe (1995) document that 30 out of 32 subjects display non-Nash behavior in a game of perfect information where such non-Nash behavior has the potential to increase payoffs to all players. Moreover, in their experiments, this sort of "trust" behavior was less prevalent when agents were in a "stronger" position. Likewise, in our experiments, we find trust behavior and we find that the prevalence of trust behavior depends on the strength of the trustee's position. For example, when insiders command a majority on a single-tiered board and thus are in a strong position, they sometimes are trustworthy, that is, they sometimes undertake actions that generate that the group-maximizing payoff. Further, when the 
insiders' position is at its weakest, as is the case in a single-tiered board with a single insider, (where all equilibria produce the minimal insider payoff) insiders are always trustworthy in that they cooperate in attaining the group-maximizing payoff.

\section{Results from Repeated Mixing Treatments}

We now present results from the four treatments employing the repeated groups protocol. We focus on (1) the incidence of the institutionally preferred outcome and (2) the predictive success of the two competing equilibria, with a view to identifying the impact of the switch from the random mixing protocol.

Table VIII contains information on the incidence of the institutionally preferred outcome. The table also contains Chi-square statistics comparing the results from each treatment with its random mixing counterpart. The only notable difference in the adoption of the institutionally preferred outcome is in the case of the single-tiered board with an insider majority. The stability of the group appears to greatly increase the frequency with which the board implements the institutionally preferred policy. The difference is significant following bad draws and appears to

be caused by statistically significantly different voting behavior by both insiders and watchdogs following bad draws.

Some Chi-square tests we conducted to examine differences in the implementation of the institutionally preferred policy across board types employing the repeated groups protocol are not reported in the tables. These tests indicate that the two single-tiered watchdog majority treatments differed significantly from the single-tiered insider majority treatment. In both cases the difference appears to be attributable to board votes following bad draws, suggesting that the stability of board composition does not make up for a lack of a watchdog majority. The Chi-square tests also confirm our earlier result with random groups that single-tiered groups with watchdog majorities adopt the institutionally preferred policy more frequently than two-tiered boards, with the difference in performance attributable to board votes following good draws.

An examination of the distribution of insider and watchdog votes reveals greater consensus among both insider and watchdog votes in the repeated treatments compared with their random grouping counterparts in which there was more evidence of insider opportunism. This finding appears to bolster our conjecture that watchdog concensus is more likely when insiders do not 
behave opportunistically.

Table IX presents evidence on the predictive success of the treatments using repeated groups. With one exception the predictive success of the competing equilibria across board types appears similar to that for the random mixing treatments. Once again the coalition-proof equilibria enjoyed limited success in the single-tiered treatments with watchdog majorities and in the two-tiered treatments. Further, efficient equilibria enjoyed the greatest success for single-tiered boards with watchdog majorities and multiple insiders. The one change from the results of the random mixing protocol treatments can be explained by the lower incidence of insider opportunism in the singletiered board treatments with insider majorities. This lower propensity for opportunistic behavior pushed the predictive success of the efficient equilibria above that of the coalition-proof equilibria for this treatment. Because the change in mixing protocols did not change in the predictive success of either set of equilibria following good draws, the improved predictive success of the efficient equilibria and the decline in the predictive success of the coalition-proof equilibria when group composition remained stable for single-tiered insider majority boards is attributable solely to changes in their performance following bad draws.

By restricting group size to seven and running the both repeated and random protocol treatments for single-tiered watchdog majority boards with multiple insiders, we are able to benchmark our results to Gillette, Noe, and Rebello (2003). We employed a Chi-square test to check for possible differences between their results and ours. The Chi-square statistic between the WMAJRAN and its counterpart in their study is 1.40 for good draws and 0.76 for bad draws. Thus, we cannot reject the null hypothesis that incidence of the institutionally preferred outcomes across the different studies' random mixing protocols are the same. The Chi-square statistic to check for differences between WMAJRE and GNR's equivalent REP treatment for good draws is 7.98 , and 0.67 for bad draws. Thus, for good draws at the 0.01 significance level, we can reject that the incidences of the institutionally preferred outcomes in the repeated group treatments are the same. Looking deeper we find that the three groups in the WMAJRE treatment of this study were always truthful and appear to have been trusted by the watchdogs in their respective groups. In Gillette, Noe, and Rebello's REP treatment just one of the three groups accounts for most of the non-institutionally preferred outcomes under the good draws. The instability of the repeated group treatment results 
we observe when comparing our sessions to Gillette, Noe, and Rebello can also be observed between our own sessions. A possible explanation for this instability for repeated group treatments is that small variations in initial interactions in the sessions have persistent effects throughout the session, leading to wide variation in outcomes across sessions.

\section{Conclusion}

In this paper we modeled and experimentally tested the performance of a variety of corporate board designs. We showed that all coalition-proof equilibria feature inefficient board decisions, biased either toward or against insider-preferred policies. However, when the board includes more than one insider, Nash equilibria exist that implement the efficient policies. When outsiders have complete control of one of the tiers on a two-tiered board or if they have majority control of a single-tiered board, these equilibria are preferred by all parties to the coalition-proof equilibria. Our experimental results showed that, despite their lack of coalition-proofness, institutionally preferred policies are adopted with considerable frequency as long as outsiders are included on the board, even when they are a minority. At the same time, increasing outsider representation to give them a majority on the board greatly increases the likelihood of the efficient outcome. Adding another tier to the board, one controlled by outsiders, was almost equally efficient in blocking the adoption of bad projects but was less successful in ensuring the implementation of good projects. When all but one insider are eliminated from a single-tiered board, efficiency is maintained despite the fact that efficient outcomes are no longer consistent with the Nash behavior.

These results have a number of implications, both practical and theoretical. First, because our results show that truly independent outside directors can act as watchdogs even if they do not have private information regarding the firm's operations, our results support the call for including more outside directors on corporate boards. Even minority outsider participation is somewhat effective in controlling managerial opportunism, especially if the outsider minority controls one tier of a two-tiered board. However, we find that outsider majority boards are most effective. This result supports the call for majority outsider representation on boards. Of course directors have roles other than being watchdogs. As Fama and Jensen (1983) point out, outside directors also provide

information complementary to insider managers' firm-specific information. Thus, in practical board design, this informational role, in addition to the watchdog role, will have to be considered in 
determining board composition. The second practical implication of our results is that two-tiered boards appear to have a conservative bias and thus may engender overly conservative investment policies and excessive hurdle rates for project adoption. This conservative bias suggests that twotiered boards are least suited for high growth firms with rich investment opportunity sets. A third practical implication of this work is that stable board composition is crucial for implementation of institutionally preferred policies only when insiders dominate the board. Thus, because there is no pressing need to guarantee board stability on boards where insiders lack control, firms may vary board composition as needed based on their changing needs for information that complements that of their managers'.

As well as providing specific practical insights, our results have some implications for mechanism design and implementation in general. First, they show that when the efficiency refinement collides with the coalition-proof refinement, efficiency seems to win out. Thus, mechanisms like ours (and the mechanisms suggested by Palfrey and Srivastava, 1993) that rely on coordination problems between agents to implement principal-preferred outcomes can work in cases where all the agents prefer the outcomes of the efficient non-coalition-proof implementing equilibria. Second, as has been documented in the experimental literature on information sharing, our results indicate that agents have a tendency to disclose information through cheap talk even when such disclosures are not in their interest. For example, in the insider majority sessions, insiders could force their preferred outcome simply by voting as a block and ignoring outsiders. Yet, in the actual play of the experiment, outsider votes were correlated with the insiders' private information. Such correlation is possible only if insiders disclose such information to the minority outsiders. Third, the differences between our results with two-tiered and single-tiered watchdog majority boards show that mechanisms with identical efficient and coalition-proof outcomes can produce different behavioral outcomes because of differences in sequencing and communication protocols. 


\section{Appendix A}

\section{A. Single-tiered Board}

This appendix provides the formal proofs of Theorems 1 through 6. Before we initiate our proofs of Theorems 1 through 5, we require some additional notation and definitions. These definitions and notation are presented below.

An agent's voting strategy is a map from observed messages into votes. Let $M^{N}$ represent a vector of messages sent by the agents, $v_{i}^{W}$ a voting strategy for watchdogs, and $v_{i}^{I}$ a voting strategy for insiders. A strategy for an individual agent is thus an ordered pair of communication and voting strategies. We represent a strategy of watchdog $i$, by $\sigma_{i}^{W} \equiv\left(m_{i}^{W}, v_{i}^{W}\right)$, and the strategy of insider

$i$, by $\sigma_{i}^{I} \equiv\left(m_{i}^{I}, v_{i}^{I}\right)$. Finally, let $\sigma^{I}$ represent the vector of insider strategies; let $\sigma^{W}$ represent the vector of watchdog strategies.

Strategies yield votes via the following functional compositions. The message strategies of the insiders and watchdogs, and the information signal, $s \in\{G, B\}$, will produce a pattern of messages. These messages will, when composed with the voting strategies of the watchdogs, produce the watchdog vote vector; when composed with insider voting strategies, they produce the insider vote vector. This process of composition generates a map, from strategies to votes, which we represent by $\mathbf{V}\left(\sigma^{W}, \sigma^{I}(s)\right)$. Using the definition of agent utility given in (1) and (2), we see that the utility of an agent $j$ under strategy vector $\sigma$ is given by

$$
u_{j}(\sigma)=\mathrm{E}\left(U_{j}\left(\mathbf{V}\left(\sigma^{W}, \sigma^{I}\right), \omega\right)\right)
$$

\section{B. Definitions}

Definition 1. For agent $j$, strategy $\sigma_{j}^{\prime}$ is a best response to $\sigma^{\prime} \in \Sigma$ if the following condition holds for all $\sigma \in \Sigma$ :

$$
\forall k \in N-\{j\}, \sigma_{k}=\sigma_{k}^{\prime} \Rightarrow u_{j}\left(\sigma^{\prime}\right) \geq u_{j}(\sigma)
$$

Definition 2. A strategy vector $\sigma^{*}$ is a Nash equilibrium if for all $j \in N, \sigma_{j}^{*}$ is a best response for $j$ to $\sigma^{*}$.

Definition 3. A coalition-proof Nash equilibrium is defined by induction on the size of coalitions as follows. Let $S \subset N$, 
(i) Suppose $\#(S)=1$, then $S=\{j\}$ for some $j \in N$. In this case, $\sigma$ is optimal for $S=\{j\}$ if and only if $\sigma_{j}$ is a best response to $\sigma$ for $j$.

(ii) Assume optimality has been defined for all $S$ such that $\#(S) \leq k-1$. Define optimality of coalitions $S$ of size $k$ as follows:

(a) $\sigma$ is self-enforcing for $S$ if $\sigma$ is optimal for $T$, whenever $T$ is a strict subset of $S$.

(b) $\sigma$ is optimal for $S$ if it is self-enforcing for $S$ and there does not exist any strategy vector $\sigma^{\prime}$ which is also self-enforcing for $S$ such that

$$
\begin{aligned}
& \forall j \in N-S, \sigma_{j}^{\prime}=\sigma_{j}, \\
& \forall j \in S, u_{j}\left(\sigma^{\prime}\right)>u_{j}(\sigma) .
\end{aligned}
$$

Finally, if $\sigma$ is optimal for $N$, we say that $\sigma$ is a coalition-proof Nash equilibrium.

Definition 4. If $\sigma$ is a strategy vector and $K$ is a subset of agents containing at least one insider, then we say that the insiders in $K$ are decisive for $\sigma$ for signal $s$ if, holding the actions of all other agents fixed, they can force acceptance of the project. In other words,

$$
\begin{aligned}
& \#(K \cap I)+\#\left\{j \in I-K: \mathbf{V}_{j}\left(\sigma^{W}, \sigma^{I}(s)\right)=\mathcal{Y}\right\}> \\
& \#\left\{j \in W: \mathbf{V}_{j}\left(\sigma^{W}, \sigma^{I}(s)\right)=\mathcal{N}\right\}+\#\left\{j \in I-K: \mathbf{V}_{j}\left(\sigma^{W}, \sigma^{I}(s)\right)=\mathcal{N}\right\}
\end{aligned}
$$

Insiders belonging to a subset of agents are decisive for a given information signal if, by collectively changing their vote to yes when they receive that signal, they can ensure that the project is accepted. Next note that all insiders have the same payoff function, and that all watchdogs have the same payoff function. Thus, the strategy vector that maximizes the payoff to a subset of agents that consists only of insiders or outsiders is well defined. This motivates the following definition.

Definition 5. Let $\sigma$ be a strategy vector, let $K$ be a nonempty "pure" subset of agents consisting only of insider types or only of outsider types. Suppose that overall strategy vectors $\sigma^{\prime}$ such that $\sigma_{j}^{\prime}=\sigma_{j}, j \notin K$, and $\sigma$ produces the highest payoff to agents in $K$, then we say that $\sigma$ maximizes payoffs over $K$.

\section{Lemma Used to Establish Theorems 1 and 2}

Our most important lemma, Lemma 1, is quite straightforward. It implies that, in coalitionproof outcomes, pure coalitions consisting of just insiders or just outsiders act as if they are a single 
agent, maximizing their collective payoff over their joint strategy space.

Lemma 1. Let $\sigma$ be a strategy vector and let $K$ be a nonempty "pure" subset of agents consisting only of insider types or only of outsider types. The strategy $\sigma$ is optimal for $K$ if and only if $\sigma$ maximizes payoffs over $K$.

Proof. Our proof is based on induction on the size of the coalition. If the coalition size, which we represent by $k$ equals one, then the lemma follows from the definition of a Nash equilibrium.

Next, suppose that Lemma 1 holds for a subset of size less than or equal to $k$. Consider a pure subset $K$ of size $k+1$ and a strategy vector, $\sigma$, that maximizes type payoffs over $K$. All subsets of a pure subset must be pure. Maximizing a type's payoff over a subset of $K$ can never yield a higher payoff than the payoff from maximizing over $K$. Thus, $\sigma$ must be self-enforcing for $k+1$. Because $\sigma$ maximizes over $K$, no other strategy vector produces a higher payoff. Thus, $\sigma$ is optimal for $K$.

To prove the other leg of the if-and-only-if assertion, suppose that $K$ is a pure coalition of size $k+1$ and let $\sigma$ be a strategy vector that does not maximize type payoffs over $K$. Then there must exist a strategy vector, say $\sigma^{\prime} \neq \sigma$, such that $\sigma^{\prime}$ equals $\sigma$ for agents not in $K$ and $\sigma^{\prime}$ maximizes the payoff over $K$. (Because there are only a finite number of distinct strategy vectors, existence of a maximizing vector is guaranteed.) By the results of the previous paragraph, $\sigma^{\prime}$ is optimal, and thus, a fortiori, self-enforcing for $K$. This implies that $\sigma$ cannot be optimal for $K$. Thus, maximization of the type payoffs over strategies in $K$ is a necessary condition for optimality as well.

By Lemma 1, insiders will force project acceptance except when project acceptance is blocked by sufficient watchdog votes. Because information regarding the information signal is transmitted only by informed insiders, insiders can always force acceptance under one signal if they can force acceptance under any signal. This reasoning underlies the next lemma, Lemma 2.

Lemma 2. If $\sigma$ is any coalition-proof Nash equilibrium, $\sigma$, the project is accepted with probability 1 or probability 0 .

Proof. Suppose that, under $\sigma$, the project is accepted under signal $s_{1}$ but not under signal $s_{2}$. Consider the subset consisting of all insiders. Consider the strategy vector $\sigma^{\prime}$ calling for insiders to use the message and voting strategy that they use when receiving $s_{1}$ under $\sigma$ for both information 
signals. Under $\sigma^{\prime}$ the project is accepted with probability 1. Assumptions (3), (4), and (5) ensure that this outcome produces a higher insider payoff than strategy $\sigma$. Thus, $\sigma$ cannot maximize the payoffs over $I$. Thus, $\sigma$ is not optimal for $I$ and thus is not coalition-proof.

Because the payoff to watchdogs is always higher if the project is rejected under both signals than it is if the project is accepted under both signals, the coalition of all watchdogs can always gain by forcing universal rejection in any candidate equilibrium in which the project is being accepted with probability 1 . Thus, such equilibria are not coalition-proof.

Lemma 3. When $w \geq i$, in any coalition-proof equilibrium, insiders are not decisive under either information signal; that is, under both signals watchdogs cast sufficient votes against the project to block passage regardless of the votes of the insiders.

Proof. Consider a coalition-proof Nash equilibrium $\sigma$. By Lemma 2 we know that if the project is accepted at all, then the project is accepted with probability 1. Thus, the equilibrium payoff to watchdogs is $\pi x(W, A, G)+(1-\pi) x(W, A, B)$. Now suppose watchdogs deviate to the strategy of always voting against the project; that is, consider the strategy vector $\sigma^{\prime}$ defined as follows. For insiders, play the strategies prescribed by $\sigma$; for outsiders, play the message strategies prescribed by $\sigma$ but follow the voting strategy of voting against the project regardless of the message sent in the message phase. Because watchdogs outnumber insiders, the project is rejected with probability 1. Thus, the strategy vector $\sigma^{\prime}$ yields watchdogs a payoff of $\pi x(W, R, G)+(1-\pi) x(W, R, B)$. By (4), this exceeds the equilibrium payoff under $\sigma$. Thus, $\sigma$ does not maximize watchdog payoffs over $W$. By Lemma 1, $\sigma$ is not optimal for $W$ and thus $\sigma$ is not coalition-proof.

Because the project is being rejected in all coalition-proof equilibria regardless of how insiders vote, and because of the penalty imposed on insiders when consensus fails, insiders collectively have an incentive to vote unanimously against project acceptance when their votes are not decisive. Thus, coalition-proof outcomes are characterized by unanimous insider rejection.

Lemma 4. When $w \geq i$, in any coalition-proof equilibrium, all insiders vote to reject the project. Proof. To obtain a contradiction, let $\sigma$ be a coalition-proof equilibrium in which not all insiders vote to reject the project. From Lemma 3 we know that in any coalition-proof equilibrium the project is voted down regardless of the insiders' voting behavior. Consider the subset of agents consisting of all insiders. If these insiders deviate to a strategy of voting against acceptance regardless of 
the messages sent in the message phase, the deviant strategy, by eliminating the possibility of the penalty for a lack of consensus, produces a higher payoff than the strategies insiders are playing under $\sigma$. Hence, $\sigma$ does not maximize payoffs for $I$. Thus, by Lemma 1, $\sigma$ is not optimal for $I$ and hence $\sigma$ is not coalition-proof.

Lemma 5. When $i>w$, in any coalition-proof equilibrium, watchdogs are not decisive under either information signal; that is, under both signals insiders cast sufficient votes in favor of the project to ensure passage regardless of the votes of the watchdogs.

Proof. Consider a coalition-proof Nash equilibrium $\sigma$. By Lemma 2 we know that if the project is rejected at all, then the project is rejected with probability 1 . Thus, the equilibrium payoff to insiders is $\pi x(I, R, G)+(1-\pi) x(I, R, B)$.

Now suppose insiders deviate to the strategy of always voting for the project; that is, consider the strategy vector $\sigma^{\prime}$ defined as follows. For watchdogs, play the strategies prescribed by $\sigma$; for insiders, play the message strategies prescribed by $\sigma$ but follow the voting strategy of voting for the project regardless of the message sent in the message phase. Because insiders outnumber watchdogs, the project is accepted with probability 1 . Thus, the strategy vector $\sigma^{\prime}$ yields insiders a payoff of $\pi x(I, A, G)+(1-\pi) x(I, A, B)$. By (4), this exceeds the equilibrium payoff under $\sigma$. Thus, $\sigma$ does not maximize payoffs for $I$. By Lemma 1, $\sigma$ is not optimal for $I$ and thus $\sigma$ is not coalition-proof.

Lemma 6. When $i>w$, in any coalition-proof equilibrium, all insiders vote to reject the project. Proof. To obtain a contradiction, let $\sigma$ be a coalition-proof equilibrium in which not all insiders vote to accept the project. From Lemma 5 we know that in any coalition-proof equilibrium the project is accepted regardless of the insiders' voting behavior. Consider the subset of agents consisting of all insiders. If these insiders deviate to a strategy of voting for acceptance regardless of the messages sent in the message phase, the deviant strategy, by eliminating the possibility of the penalty for a lack of consensus, produces a higher payoff than the strategies insiders are playing under $\sigma$. Hence, $\sigma$ does not maximize payoffs for $I$. Thus, by Lemma 1, $\sigma$ is not optimal for $I$ and hence $\sigma$ is not coalition-proof.

Lemmas 1 to 6 characterize coalition-proof equilibria. We now turn our attention to proving that coalition-proof equilibria exist. The existence proof requires us to consider mixed insider- 
watchdog coalitions. Characterizing such coalitions motivates the following definitions.

Definition 6. A coalition of agents, $K$, is flawed under strategy vector $\sigma$, if there exists an information signal $s$ such that the following conditions hold.

a. The project is rejected under $s$; that is, for some $s$,

$$
\# \mathcal{Y}\left(\mathbf{V}\left(\sigma^{W}, \sigma^{I}(s)\right)\right) \leq \# \mathcal{N}\left(\mathbf{V}\left(\sigma^{W}, \sigma^{I}(s)\right)\right)
$$

b. The coalition $K$ contains at least one insider.

c. The insiders in $K$ are decisive for $\sigma$ under $s$.

A flawed coalition contains a subset of insider agents who are decisive for project acceptance yet fail to ensure that the project is always accepted. In the subsequent analysis we will show that equilibria in which the set of all agents is flawed are not coalition-proof. Because coalition-proofness is defined by induction on subset size, we must define flawed coalitions not only for the set of all agents, but also for all proper subsets of agents. The next lemma shows that, when the strategy vector is flawed by collective changes in their strategy, a sufficiently large coalition of insiders can always modify their strategies to ensure project acceptance.

Lemma 7. If $J$ is flawed for $\sigma$ and if all insiders not in $J$ are sending the same message under both signals, there exists a strategy vector, $\sigma^{\prime}$, which specifies the same strategies as $\sigma$ for all watchdogs and insiders not in $J$ such that the project is accepted with probability 1.

Proof. We construct the strategy vector as follows. Let $K=I \cup J$, for all agents not in $K$; let $\sigma^{\prime}=\sigma$. Next, determine the signal, say $s^{\prime}$, under which insiders are decisive. Each agent in $K$ should (a) send under both signals the message that, under $\sigma$, she sent under $s^{\prime}$, and (b) subsequently vote to accept the project regardless of the pattern of messages received. This strategy will ensure that the project is accepted under both signals with the unanimous support of insiders in $K$.

Lemma 8. If $J$ is flawed for $\sigma$ and if all insiders not in $J$ are sending the same message under both signals, then $\sigma$ is not optimal for $J$.

Proof. By Lemma 6, $\sigma$ does not maximize the payoff over $I \cup J$. Thus, by Lemma $1, \sigma$ is not optimal for $J$.

Lemma 9. When $w \geq i$, there exists a coalition-proof Nash equilibrium under which the project is rejected with probability 1 and all insiders cast votes against project acceptance under both information signals. 
Proof. Consider the strategy vector $\sigma$ defined as follows. All agents send the same arbitrary message, $m_{0}$, independent of the information signal, that is $\mathbf{m}_{i}^{W}=\mathbf{m}_{i}^{I}(s)=m_{0}$. All insiders and watchdogs follow the strategy of voting $\mathcal{N}$ regardless of the information signal, that is, $\mathbf{v}_{i}^{W}(m)=\mathcal{N}$, $\mathbf{v}_{i}^{I}(m, G)=\mathcal{N}, \mathbf{v}_{i}^{I}(m, B)=\mathcal{N}$. To show that this is a coalition-proof Nash equilibrium, we need to show that $\sigma$ is optimal for all subsets of $N$. To show this we provide a proof by induction on subset size. First note that when the subsets contain a single element, optimality simply requires that for all agents $j, \sigma_{j}$ is a best response to $\sigma$ for $j$. However, no individual agent can change the project acceptance decision by unilaterally changing his strategy. Moreover, insiders may call down a penalty if they deviate from the consensus. Thus, the assertion of optimality holds for all subsets of size one. Next suppose that, for subsets of size less than or equal to $k$, optimality holds. Consider a subset $K$ of size $k+1$. Given the induction hypotheses and the definition of coalition-proofness, optimality requires that there does not exist another self-enforcing strategy for $K$ that yields all the agents in $K$ a higher payoff. If $K$ consists only of watchdogs, $K$ cannot produce a higher payoff because, given the uninformative messages of insiders, watchdogs cannot induce a strategy vector that accepts the project under the good information signal and rejects the project under the bad signal. Given assumptions (3), (4), and (5), rejecting the project under both signals produces a higher payoff to watchdogs than accepting the project under both signals. No improving vector of strategies exists for $K$, and thus, a fortiori, no self-enforcing vector of strategies exists for $K$ when $K$ consists of a set of watchdogs. Next note that a coalition of all insiders does not have sufficient votes to change the outcome. Thus, such a subset cannot increase its welfare by deviating from the equilibrium. Only a mixed coalition, by implementing a vector of strategies calling for rejection when the information signal is $B$ and acceptance when the information signal is $G$, can increase the payoff to all agents in $K$. However, by our earlier definition, such a coalition is flawed. Lemma 6 shows that a flawed coalition is not optimal and thus, a fortiori, is not self-enforcing. Thus, optimality for coalitions of size $k+1$ has been established, proving the assertion of the theorem by induction.

Lemma 10. When $i>w$, there exists a coalition-proof Nash equilibrium under which the project is accepted with probability 1 and all insiders vote for project acceptance under both information signals. 
Proof. Consider the strategy vector $\sigma$ defined as follows. All agents send the same arbitrary message, $m_{0}$, independent of the information signal, that is, $\mathbf{m}_{i}^{W}=\mathbf{m}_{i}^{I}(s)=m_{0}$. All insiders follow the strategy of voting $\mathcal{Y}$ regardless of the information signal, that is, $\mathbf{v}_{i}^{I}(m, G)=\mathcal{N}, \mathbf{v}_{i}^{I}(m, B)=\mathcal{N}$. All watchdogs abstain, that is $\mathbf{v}_{i}^{W}(m)=\mathcal{A}$. To show that this is a coalition-proof Nash equilibrium, we need to show that $\sigma$ is optimal for all subsets of $N$. To show this we provide a proof by induction on subset size. First note that when the subsets contain a single element, optimality simply requires that for all agents $j, \sigma_{j}$ is a best response to $\sigma$ for $j$. However, no individual agent can change the project acceptance decision by unilaterally changing his strategy. Moreover, insiders may call down a penalty if they deviate from the consensus. Thus, the assertion of optimality holds for all subsets of size one. Next suppose that, for subsets of size less than or equal to $k$, optimality holds. Consider a subset $K$ of size $k+1$. Given the induction hypotheses and the definition of coalition-proofness, optimality requires that there does not exist another self-enforcing strategy for $K$ that yields all the agents in $K$ a higher payoff. If $K$ consists only of insiders, $K$ cannot produce a higher payoff because given assumptions (3), (4), and (5), accepting the project under both signals produces the highest payoff to insiders. Next note that a coalition of all watchdogs does not have sufficient votes to change the outcome. Thus, such a subset cannot increase its welfare by deviating from the equilibrium. There also exists no self-enforcing strategy for a mixed coalition to deviate from the equilibrium as any insiders in the deviating coalition will be strictly worse off than they are under the equilibrium. Thus, optimality for coalitions of size $k+1$ has been established, proving the assertion of the lemma by induction.

Proof of Theorem 1. The proof of the part of the first claim that all insiders vote to accept the project under both information signals, watchdog votes are unrestricted, and the project is accepted regardless of the information signal, follows directly from Lemmas 5 and 6 . The existence of coalition-proof equilibria follows from Lemma 10.

Proof of Theorem 2. First we prove the claim that in all Nash equilibria that implement the efficient outcome all insiders vote $\mathcal{Y}$ if they observe the signal $G$ and vote $\mathcal{N}$ if they observe the signal $B$. To see this, note that consensus among insiders is necessary in all Nash equilibria that implement the efficient outcome. This follows because a lack of unanimity among insiders would result in the possibility that insiders would bear a penalty. 
Next we show that an equilibrium exists that implements the efficient outcome. The equilibrium is given as follows. All agents send the same arbitrary message $m_{0}$, that is, $m_{i}^{W}=m_{i}^{I}(s)=$ $m_{0}$. All insiders follow the strategy of voting $\mathcal{Y}$ if and only if they receive information signal $G$; $v_{i}^{I}(m, G)=\mathcal{Y}, v_{i}^{I}(m, B)=\mathcal{N}$. All watchdogs abstain regardless of the messages they observe, $v_{i}^{W}(m)=\mathcal{A}$. In this candidate equilibrium, the vote of an individual agent cannot change the project selected. Moreover, for insiders, deviation from the strategy may incur the penalty for lack of consensus. Thus, unilateral deviations from the candidate information strategy vector cannot increase the payoff to any of the agents. It follows that the candidate strategy vector is a Nash equilibrium.

Proof of Theorem 3. Suppose that a Nash equilibrium exists that implements the efficient outcome. Let the strategy vector supporting this equilibrium be $\sigma$. Now consider the strategy vector $\sigma^{\prime}$. Let ${\sigma^{\prime}}_{j}^{w}=\sigma_{j}^{w}$ for all $j \in W$. For the sole insider, let ${\sigma^{\prime}}_{k}^{i}(B)={\sigma^{\prime}}_{k}^{i}(G)=\sigma_{k}^{i}(G)$; that is, under $\sigma^{\prime}$ the insider sends the same message and votes the same way as he would following the observation of signal $G$ under strategy $\sigma$. It follows that the project will be accepted regardless of the signal. Further, from assumption (3), the insider is strictly better off under $\sigma^{\prime}$. It follows then that $\sigma$ could not support a Nash equilibrium.

Proof of Theorem 4. The proof of the part of the first claim that the insider votes to reject the project under both information signals, at least one watchdog votes to reject at all times, and the project is rejected regardless of the information signal, follows directly from Lemmas 3 and 4 . The existence of coalition-proof equilibria follows from Lemma 9.

Proof of Theorem 5. The proof of (i) is identical to that of Theorem 4. The proof of the claim that the insider votes to reject the project under both information signals, at least one watchdog votes to reject at all times, and the project is rejected regardless of the information signal, follows directly from Lemmas 3 and 4 . The existence of coalition-proof equilibria follows from Lemma 9.

The proof of (ii) is identical to the proof of Theorem 2. First we prove the claim that, in all Nash equilibria that implement the efficient outcome, all insiders vote $\mathcal{Y}$ if they observe the signal $G$ and vote $\mathcal{N}$ if they observe the signal $B$. To see this, note that consensus among insiders is necessary in all Nash equilibria that implement the efficient outcome. This follows because a lack of unanimity among insiders would result in the possibility that insiders would bear a penalty. 
Next we show that an equilibrium exists that implements the efficient outcome. The equilibrium is given as follows. All agents send the same arbitrary message $m_{0}$, that is, $m_{i}^{W}=m_{i}^{I}(s)=$ $m_{0}$. All insiders follow the strategy of voting $\mathcal{Y}$ if and only if they receive information signal $G$; $v_{i}^{I}(m, G)=\mathcal{Y}, v_{i}^{I}(m, B)=\mathcal{N}$. All watchdogs abstain regardless of the messages they observe, $v_{i}^{W}(m)=\mathcal{A}$. In this candidate equilibrium, the vote of an individual agent cannot change the project selected. Moreover, for insiders, deviation from the strategy may incur the penalty for lack of consensus. Thus, unilateral deviations from the candidate information strategy vector cannot increase the payoff to any of the agents. It follows that the candidate strategy vector is a Nash equilibrium.

\section{Two-tiered Board}

Before we initiate our proof of Theorem 6 , we require some additional notation and definitions as the communication and voting sequence for a two-tiered board is different from that of a singletiered board. These definitions and notation are presented below.

An agent's voting strategy is a map from observed messages into votes. Let $v_{i}^{W}$ represent a vote by watchdog $i$ and $v_{i}^{I}$ a vote for insider $i$. Given that they communicate with each other and cast their votes based on messages exchanged among insiders alone, a strategy for an insider is thus an ordered pair of messages and votes. We represent a strategy for insider $i$ by $\sigma_{i}^{I} \equiv\left(m_{i}^{I}, v_{i}^{I}\right)$. Finally, let $\sigma^{I}$ represent the vector of insider strategies.

Note that the supervisory board receives a report from a representative of the executive board following a majority yes vote by the executive board. ${ }^{8}$ Let this event be represented by $\sigma^{R} \in \Sigma^{R}$, where $\Sigma^{R} \equiv\left\{\left(\sigma^{I}, m^{R}\right): \mathcal{Y}\left(v^{I}\right)>\mathcal{N}\left(v^{I}\right)\right\}$ and $m^{R}$ is a function mapping the results of the insider voting into a report. Given that they vote after insiders vote to accept, watchdog strategies are conditional on $\sigma^{R}$.

Given the sequence of voting and communication, we restrict attention to subgame perfect Nash equilibria and coalition-proof equilibria where coalition formation is subgame perfect, that

8 Given that all insiders are identical and receive the same payoff, it does not matter which insider presents this report to the supervisory board. 
is, coalitions are formed only among members of the same board tier.

Proof of Theorem 6. Because coalition formation is subgame perfect, coalitions can be formed only between agents with identical payoffs. Thus, an equilibrium is coalition-proof in this sense if and only if it is a Nash equilibrium that maximizes the aggregate payoff of each agent type. Thus, it is easy to see that no equilibrium exists in which the project is ever accepted. Suppose that such an equilibrium existed, then there would be a vote and message combination for insiders that induces project acceptance and thus insiders would have incentive and ability to always send this message and vote vector. It follows that the project would always be accepted. However, if the project were always accepted, watchdogs would maximize their aggregate payoff by rejecting the project.

The proof of (ii) is identical to the proof of Theorem 2. First we prove the claim that, in all Nash equilibria that implement the efficient outcome, all insiders vote $\mathcal{Y}$ if they observe the signal $G$ and vote $\mathcal{N}$ if they observe the signal $B$. To see this, note that consensus among insiders is necessary in all Nash equilibria that implement the efficient outcome. This follows because a lack of unanimity among insiders would result in the possibility that insiders would bear a penalty.

Next we show that an equilibrium exists that implements the efficient outcome. The equilibrium is given as follows. All agents send an arbitrary message at each stage of the game (including the report to the supervisory board). All insiders follow the strategy of voting $\mathcal{Y}$ if and only if they receive information signal $G ; v_{i}^{I}(m, G)=\mathcal{Y}, v_{i}^{I}(m, B)=\mathcal{N}$. All watchdogs abstain regardless of the messages and report they observe $v_{i}^{W}(m)=\mathcal{A}$. In this candidate equilibrium, the vote of an individual agent cannot change the project selected. Moreover, for insiders, deviation from the strategy may incur the penalty for lack of consensus. Thus, unilateral deviations from the candidate information strategy vector cannot increase the payoff to any of the agents. It follows that the candidate strategy vector is a Nash equilibrium. 


\section{REFERENCES}

Berg, J., J. Dickhaut, and K. McCabe, 1995, Trust, reciprocity, and social history, Games and Economic Behavior 10, 122-142.

Bernheim, D., B. Peleg, and M. Whinston, 1987, Coalition-proof Nash equilibria I: Concepts, Journal of Economic Theory 42, 1-12.

Bhattacharya, U., and H. Daouk, 2002, The world price of insider trading, Journal of Finance 57, $75-108$.

Camerer, C., 2003, Behavioral Game Theory Experiments in Strategic Interaction, Princeton, NJ: Princeton University Press.

Coles, J., M. Lemmon, and J. Meschke, 2003, Structural models endogeneity in corporate finance, Arizona State University working paper.

Cosmides, L. and J. Tooby, 1989, Evolutionary Psychology and the Generation of Culture, Part II: Case Study: A Computational Theory of Social Exchange, Ethology and Sociobiology $10,51-9$.

Davis, D. and C. Holt, 1992, Experimental Economics, Princeton: Princeton University Press.

DeJong, D., R. Forsythe, and W. Uecker, 1988, A Note on the Use of Businessmen as Subjects in Sealed Offer Markets, Journal of Economic Behavior and Organization, 9, 87-100.

Dyer, D., J. Kagel, and D. Levin, 1989, A Comparison of Nave and Experienced Bidders in Common Value Offer Auctions: A Laboratory Analysis, Economic Journal, 99, 108-115.

Economist, 2003, Thin on top, January 23.

Eckel, C., and C. Holt, 1989, Strategic Voting and Agenda-Controlled Committee Experiments, American Economic Review 79, 763-773.

Fama, E. and M. Jensen, 1983, Separation of Ownership and Control, Journal of Law and Economics 26, 301-325.

Fehr, E., G. Kircksteiger, A. Reidl, 1993, Does Fairness Prevent Market Clearing? An Experimental Investigation, Quarterly Journal of Economics 108, 437-459..

Forsythe, R., J. Horowitz, N. Savin, and M. Sefton, 1991, Fairness in simple bargaining experiments, Games and Economic Behavior 6, 347-369.

Franks, Julian, Colin Mayer, and Stefano Rossi, 2004, Ownership: Evolution and regulation, ECGI - Finance Working Paper No. 09/2003.

Gillette, A., T. Noe, and M. Rebello, 2003, Corporate Board Composition, Protocols, and Voting Behavior: Experimental Evidence, Journal of Finance 58, 1997-2031.

Gneezy U. and A. Rustichini A, 2000, Pay Enough or Don't Pay At All, The Quarterly Journal of Economics 115, 791-810.

Hermalin, B. and M. Weisbach, 2001, Boards of Directors as an Endogenously Determined Institution: A survey of the Economic Literature, N.B.E.R. working paper.

Hermalin, Benjamin, and Michael Weisbach, 1991, The Effects of Board Composition and Direct Incentives on Firm performance, Financial Management 20, 101-112. 
Hoffman, E., K. McCabe. and V. Smith, 1996. On Expectations and the Monetary Stakes in Ultimatum Games International Journal of Game Theory 25, 289-301.

Issac, M., and V. Smith, 1985, In Search of Predatory Pricing, Journal of Political Economy, 93, $320-345$.

Kahn, Charles and Andrew Winton, 1998, Ownership Structure, Speculation, and Shareholder Intervention, Journal of Finance 53, 99-129.

Klein, A., 2002, Audit Committee, Board of Director Characteristics and Earnings Management Journal of Accounting and Economics 33, 375-400.

Ledyard, J., 1995, Public Goods: A Survey of Experimental Research, in Handbook of Experimental Economics, J. Kagel and A. Roth (eds.) Princeton: Princeton University Press.

Mestelman, S. and D. Feeny, 1988, Does Ideology Matter?: Anecdotal Experimental Evidence on the Voluntary Provision of Public Goods, Public Choice, 57, 281-286.

Palfrey, Thomas R., and Sanjay Srivastava, 1993, Bayesian implementation, Chur, Switzerland: Harwood Academic Publishers.

Plott, C., 1987, Dimensions of Parallelism: Some Policy Applications of Experimental Methods, in Laboratory Experimentation in Economics: Six Points of View, Cambridge: Cambridge University Press.

Plott, C. , 1988, Research on pricing a Gas Transportation Network, Office of Economic Policy Technical Report No. 88-2. Federal Energy Regulatory Commission, Washington, DC.

PricewaterhouseCoopers, 2002, Bundesverband der Deutchen industrie

Raheja, Charu, 2004, Determinants of Board Size and Composition, Journal of Financial and Quantitative Analysis, forthcoming.

Selten, R., 1991, Properties of a Measure of Predictive Success, Mathematical Social Sciences 21, 153-167.

Smith, V., 1976, Experimental Economics: Induced Value Theory, American Economic Review Papers and Proceedings, 66, 274-279.

Smith, V., 1982, Microeconomic Systems as an Experimental Science, American Economic Review, $72,923-955$.

Smith, V., G. Suchanek, and A. Williams, 1988, Bubbles, Crashes, and Endogenous Expectations in Experimental Spot Asset Markets, Econometrica, 56, 1119-1151.

Tooby, J., and L. Cosmides, 1992, The Psychological Foundations of Culture in The Adapted Mind: Evolutionary Psychology and the Generation of Culture, J. Barkow, L. Cosmides, and J. Tooby (eds.) New York: Oxford University Press.

Valley, K., L. Thompson, R. Gibbons, and M. Glazerman, 2002, How Communication Improves Efficiency in Bargaining Games, Games and Economic Behavior, 38, 127-155. 